\title{
Spur gear fault diagnosis using FIR and IIR Filter
}

\author{
Sanjeev Kumar ${ }^{1}$, Ghanshyam Das ${ }^{2}$ \\ Assistant Professor, Dept of Mechanical Engg, SRM University, NCR Campus, Modinagar, Uttar Pradesh, India ${ }^{1}$
}

Assistant Professor, Department of Mechanical Engineering, BBDIT, Ghaziabad, Uttar Pradesh, India ${ }^{2}$

\begin{abstract}
The main objective of the work carried out in this paper was to study the response of messing tooth wear on vibration signal and suggest a suitable signal processing technique which can help in detecting the defects easily. Experiments were conducted on meshing gear assembly fabricated for the purpose. The signals were acquired using both the accelerometer and acoustic sensors. It has been observed that the wavelet de-noising is more effective for filtering of defective gear vibration signal compared to standard FIR and IIR filter. Part of the signal representing defect becomes more prominent after wavelet de-noising. It results in the kurtosis parameter improved twice with respect to raw signal after filtering. For the vibration signal processing, the noise reduces by $42 \%$ which justifies the selection of wavelet filtering. In the wavelet de-noising, the vibration burst was not getting distorted during filtering for vibration. The interpretation of spikes from enveloped signal with driver gear rotation was easier to understand and correlate for the cases, where vibration burst was present in vibration signal due to defect. In the case when the vibration burst is suppressed by other noise or is having low amplitude, undecimated wavelet transform implementation was more suitable. It shows that UDT also acts as a de-noising tool for gear vibration signal analysis. In vibration signal processing, the approximation signal was having higher Kurtosis value and lesser noise. For the acoustic signal processing, Kurtosis parameter improved $70 \%$ with respect to raw signal after filtering. The approximation signal having- little higher kurtosis and SNR values than raw signal, but detail coefficients were having significant higher kurtosis value and lower SNR value.
\end{abstract}

Keywords: MATLAB, LABVIEW, Data acquisition system, Accelerometer.

\section{INTRODUCTION}

A reliable online condition monitoring system is very useful in industries to provide accurate fault diagnostic information in mechanical systems to prevent machinery performance degradation, malfunction or even catastrophic failures. On the other hand, machinery condition monitoring information can also enable the establishment of a maintenance program based on an early warning of incipient defects. Fault diagnosis is a sequential process involving two steps: representative feature extraction and pattern classification. Feature extraction is a mapping process from measured signal space to the feature space. Representative features associated with the health condition of a machinery component (or subsystem) are extracted by using appropriate signal processing techniques. Vibration signals are used for analysis in this case [Wilson Wang, 2009]. Diagnostics is based on the characterization of mechanical system condition and allows early detection of a possible fault. Whatever the mechanical system, the evaluation of both the type and the fault position allows in a reduction of the plant standstill time. Therefore, from an industrial point of view, a proper diagnostics approach reduces both the time and the costs required for repairing. These considerations have encouraged investments of resources in the diagnostic field. Signal processing is an approach widely used in diagnostics, since it allows directly characterizing the state of the system. Several types of advanced signal processing techniques have been proposed in the last decades and added to more conventional ones. Since each technique is based on different theoretical background, also the results obtained are often different. Some techniques may be more suitable than others may for a specific system or component, depending also on the environmental conditions. Therefore, it is important to choose techniques that are the most effective for the case and the situation under testing for a reliable mechanical analysis [Roberto Ricci, 2010]. Unlike the other monitoring techniques, vibration monitoring is a generically well-suited technique and is hence widely used to detect, locate, and diagnose failures in rotating machinery. Gearboxes are widely used in industry to transmit power or rotary motion whilst maintaining an intended torque and angular velocity ratio together with smooth motion and high efficiency. During normal operation, the components of a gearbox are subjected to dynamic loads, which cause a variety of responses including stress, deformation, changes in temperature and vibration. The characteristics of these responses, in particular those of vibration, change when a fault occurs. A gear set may exhibit a variety of failure modes depending upon the operating conditions and gear properties. Each failure mode will generally change the gearbox vibration characteristics in its own particular way, making possible the detection and classification of the fault. It is for this reason that vibration-based condition monitoring is widely used in gearbox fault detection and diagnosis. The monitored signal to be analyzed influences the type of analysis to be performed and conventional 
methods can be used to define the characteristics of stationary signals. However, in many cases the spectral composition of gearbox vibration changes with time, meaning that Fourier analysis cannot truly reflect such vibration characteristics. One approach to non-stationary signal analysis is combined time-frequency representation, which offers an alternative way of representing signals by presenting simultaneously information in the time and frequency domains, and which can be performed with either constant or varying time-frequency resolution [Isa Yesilyurt, 2003].

Aiwina Heng communicated his review article first synthesizes and places these individual pieces of information in context, while identifying their merits and weaknesses. It then discusses the identified challenges, and in doing so, alerts researchers to opportunities for conducting advanced research in the field. Current methods for predicting rotating machinery failures are summarized and classified as conventional reliability models, condition-based prognostics models and models integrating reliability and prognostics. Areas in need of development or improvement include the integration of condition monitoring and reliability, utilization of incomplete trending data, consideration of effects from maintenance actions and variable operating conditions, derivation of the non-linear relationship between measured data and actual asset health, consideration of failure interactions, practicability of requirements and assumptions, as well as development of performance evaluation frameworks [Aiwina Heng, 2009, x].

\section{EXPERIMENTAL SET UP}

An experiment were also conducted by loading the driven shaft by different weights and was found that $04 \mathrm{~kg}$ load as a suitable one to carry out the experimentation with proper loading along with dynamic stability of the system. The data from accelerometer were captured with National Instrument's SCXI system through a Lab view 7.1 based programs. The program displays the raw signal and its Fourier Transform on the screen of computer monitor. The program has facility to vary the sampling rate and display the multiple acquisitions from up to four sensors at a time. We have acquired the signal at sampling rate 70000 samples per second. Acoustic emissions were captured through mike and sound card of the computer. Components of data acquisition system used for the experiment is shown in Fig.1.

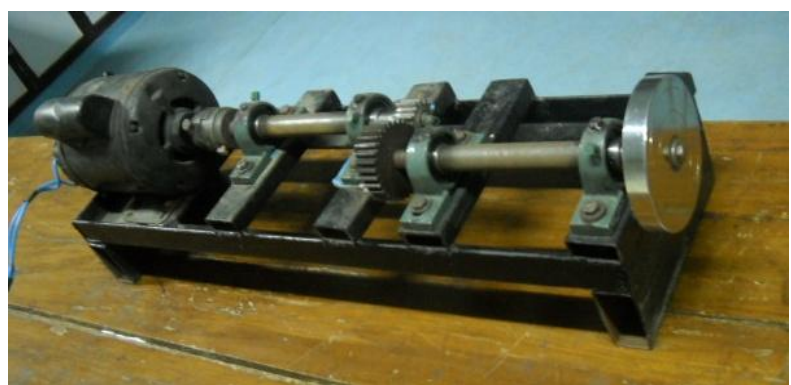

Fig .1 Photograph of experimental setup
Operating frequency of the shaft is observed as $24.8 \mathrm{~Hz}$ (i.e. approx. $1485 \mathrm{rpm}$ ). Third to sixth harmonics of operating frequency are prominent even without the gears in mesh.

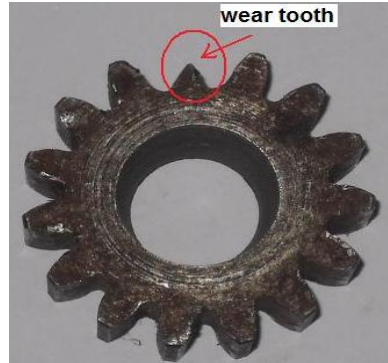

(a)

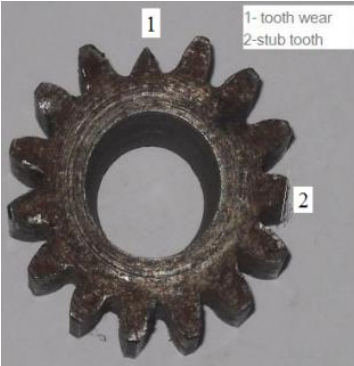

(b)
Fig. 2: Defective driver gear images (a) Photograph of pinion having one wear tooth, (b) Photograph of pinion having one wear tooth

\section{GEAR TOOTH FAILURE}

The vibration and acoustic signal for gear having one tooth failure was used for selecting suitable filtering technique so as to identify the busts in the signal prominently. The filtering technique was evaluated for vibration and acoustic signal separately.

\section{IV.VIBRATION SIGNAL PROCESSING}

In first phase the suitable filters for vibration signal were evaluated using the vibration signal for single teeth failure. Firstly the standard filters were implemented on vibration signals, i.e. FIR, IIR high pass and band pass filters. Further wavelet denoising was tested. The various filter definitions were tabulated in below table 1 .

\section{TABLE 1: FILTER TYPES AND DETAILS APPLIED FOR FILTERING}

\begin{tabular}{|l|l|}
\hline Filter-1 & $\begin{array}{l}\text { IIR Butterworth Filter (Low pass 20K } \\
\text { Hz) of order-5 }\end{array}$ \\
\hline Filter-2 & $\begin{array}{l}\text { IIR Butterworth Filter (Band pass 20-20K } \\
\text { Hz) of order-5 }\end{array}$ \\
\hline Filter-3 & FIR Filter (Low pass 20K Hz) of 29 taps, \\
\hline Filter-4 & $\begin{array}{l}\text { FIR Filter (Band pass 20-20K Hz) of 29 } \\
\text { taps }\end{array}$ \\
\hline Filter-5 & $\begin{array}{l}\text { Rectangular smoothing filter (half width } \\
\text { of moving average) }\end{array}$ \\
\hline Filter-6 & $\begin{array}{l}\text { Wavelet denoised filter (db-4 wavelet, } \\
\text { level-3, DWT transform, multiple re- } \\
\text { scaling, SURE thresholding) }\end{array}$ \\
\hline
\end{tabular}

(a) Wavelet denoising: The wavelet threshold de-noising procedure has three steps. In first phase transform the vibration signal to the time-scale plane by means of a wavelet transform. It is possible to acquire the results of the wavelet coefficients on different scales. In second phase assess the thresholding technique and thresholding rule (to shrink the wavelet coefficients), followed by using 
the shrunken coefficients to carry out the inverse wavelet transform in third phase. The series recovered is the estimation of impulse. In current analysis the raw vibration signal was decomposed using DWT (discrete wavelet transform) up to level three using db-4 as mother wavelet. The hard thresholding was implemented in all levels using SURE thresholding rule. The reconstruction of signal was carried out by inverse wavelet transform. The reconstructed signal was denoised signal, shown in figure 3 . In the figure the denoised signal was overlapped with raw signal and justifies the filtering.

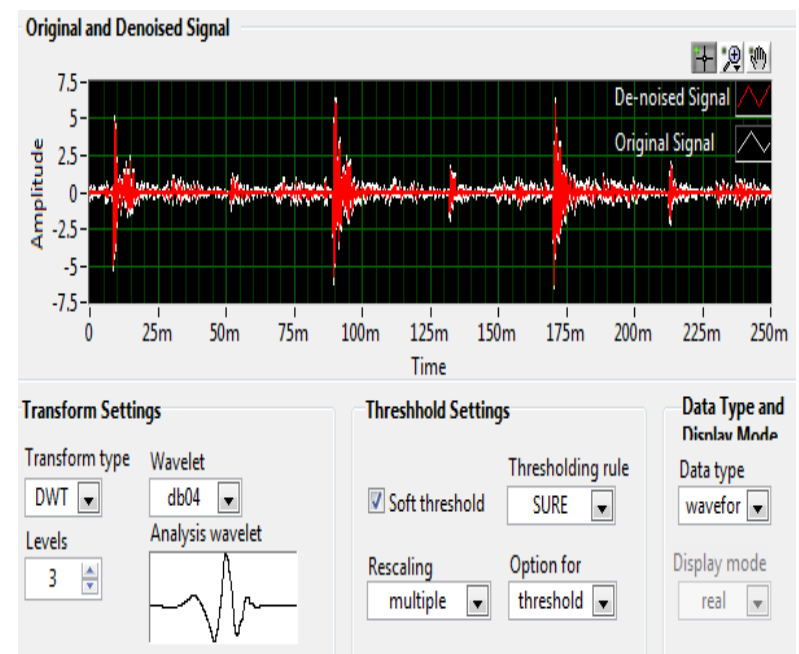

Fig.3: GUI of wavelet based denoising; using DWT technique, db-4 as mother wavelet, level of decomposition

3, hard thresholding using SURE threshold rule

(b) Enveloping of signal: Enveloping of signal extracted the modulating signal or envelope signal from an amplitude-modulated signal. Envelope detection technique was implemented to identify the mechanical faults that have amplitude-modulating effect on the vibration signal of a machine.

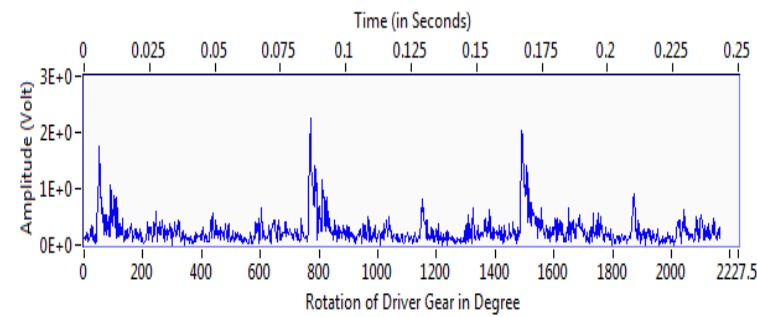

(a) Envelope of raw signal

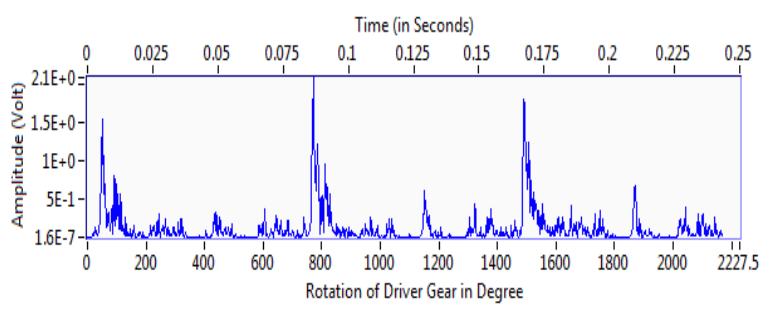

(b) Envelope of wavelet denoised signal

Fig. 4: Enveloping of signal; (a) envelop of raw vibration signal, (b) enveloping of wavelet denoised vibration signal
GEAR TEETH FAILURE (two teeth defective)

Vibration signal processing
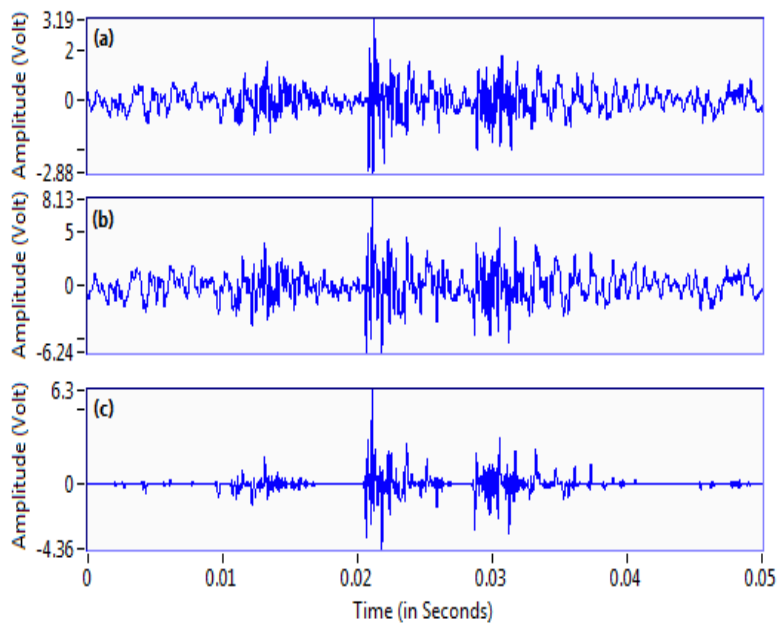

Fig.5: Raw signal and different steps of filtering (a) Raw acoustic signal burst due to2- teeth defect, (b) Approximation at level-3 using UWT, (c) Denoised signal using DWT

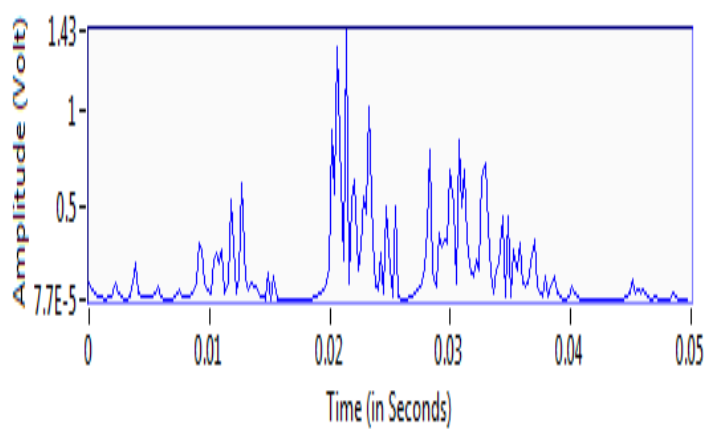

Fig. 6: Envelope of wavelet based denoised signal shown in figure $5(\mathrm{c})$
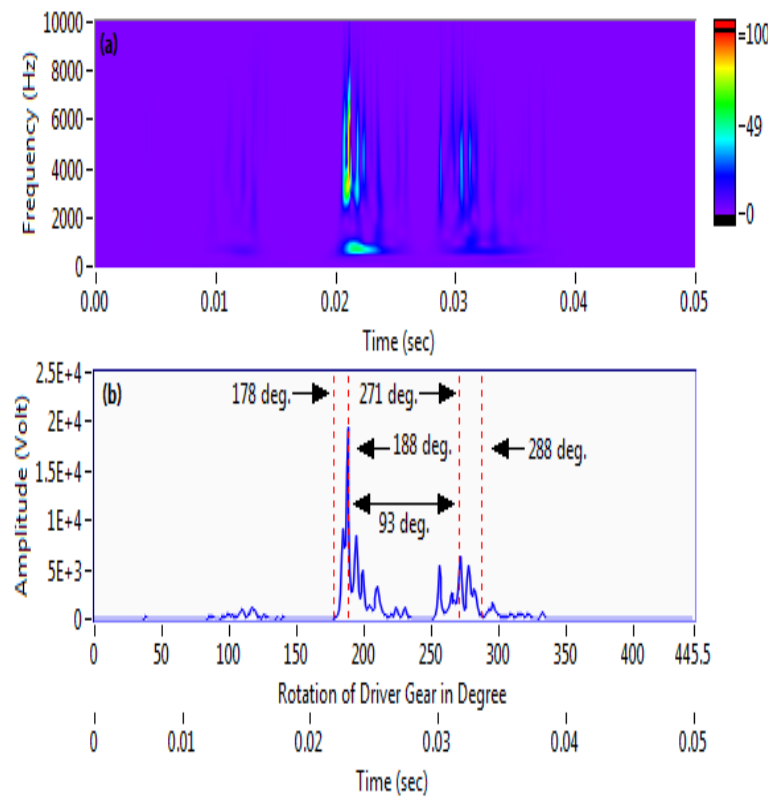

Fig.7: Analytical wavelet transform of a sample filtered signal and TMI of coefficients (a) AWT coefficient spectrum and (b) TMI of AWT coefficients 


\section{CONCLUSION}

- Part of the signal representing defect becomes more prominent after wavelet de-noising. It results in the kurtosis parameter improved twice with respect to raw signal after filtering. For the vibration signal processing, the noise reduces by $42 \%$ which justifies the selection of wavelet filtering. In the wavelet denoising, the vibration burst was not getting distorted during filtering for vibration signal as well as acoustic signal processing.

- The interpretation of spikes from enveloped signal with driver gear rotation was easier to understand and correlate for the cases, where vibration burst was present in vibration signal due to defect.

- In the case when the vibration burst suppressed is suppressed by other noise or is having low amplitude, undecimated wavelet transform implementation was more suitable. It shows that UDT also acts as a denoising tool for gear vibration signal analysis.

\section{REFERENCES}

[1] H. Zheng, Z. Li and X. Chen, "Gear Fault Diagnosis Based on Continuous Wavelet Transform”, Mechanical Systems and Signal Processing, vol. 16 (2-3), pp. 447-457, 2002.

[2] Hoonbin Hong, and Ming Liang, "Fault severity assessment for rolling element bearings using the Lempel-Ziv complexity and continuous wavelet transform", Journal of Sound and Vibration, vol. 320, pp. 452-468, 2009.

[3] Isa Yesilyurt, "Fault detection and location in gears by the smoothed instantaneous power spectrum distribution", NDT \& E International, vol. 36, pp. 535-542, 2003.

[4] I. Soltani Bozchalooi, Ming Liang, "A joint resonance frequency estimation and in-band noise reduction method for enhancing the detectability of bearing fault signals", Mechanical Systems and Signal Processing, vol. 22, pp. 915-933, 2008.

[5] Jafar Zarei, and Javad Poshtan, "An advanced Park's vectors approach for bearing fault detection", Tribology International, vol. 42, pp. 213-219, 2009.

[6] J. Antoni, "Cyclic spectral analysis of rolling-element bearing signals: Facts and fictions", Journal of Sound and Vibration, vol. 304, pp. 497-529, 2007.

[7] J. Rafiee, M.A. Rafiee, and P.W. Tse, "Application of mother wavelet functions for automatic gear and bearing fault diagnosis", Expert Systems with Applications, vol. 37, pp. 4568-4579, 2010.

[8] James I. Taylor, "The vibration analysis hand book", First edition second printing.

[9] J. Rafiee, and P. W. Tse, "Use of autocorrelation of wavelet coefficients for fault diagnosis", Mechanical Systems and Signal Processing, vol. 23, pp. 1554-1572, 2009. 